\title{
Towards safer methods of electronic surveillance
}

\author{
Mark M McGilchrist \\ University of Dundee \\ Mackenzie Building, \\ Kirsty Semple Way, \\ Dundee, DD2 4BF \\ m.m.mcgilchrist@dundee.ac.uk
}

\begin{abstract}
Surveillance activities by governments and corporations are at the forefront of information governance concerns. However, these activities extend into many other sectors such as health and social care and are a necessary part of the information society. It is essential that they are conducted in a safe manner. Most implementations involve centralisation of data and activities and a high degree of trust is demanded. However, distributed approaches are possible and could be much safer. An approach is described and briefly evaluated using a study within the health and social care domain.
\end{abstract}

Surveillance, democracy, information society, distributed architecture, record linkage.

\section{INTRODUCTION}

Few object to the notion that we are progressing towards an information society. Many however, would argue over its defining characteristics. One good candidate is that of population surveillance, leading to new knowledge that can influence behaviour at various scales. It is the one that raises most concern, centred principally on the threat to privacy and confidentiality. But other issues matter, including threats to democracy, and monopolistic economic behaviour.

This paper accepts this fundamental conception of the information society and offers the following operational definition: A society which uses information technology and controlled data flows to achieve greater self-understanding and adaptability of its politics, economy, society and culture.

In the popular imagination surveillance appears to be concentrated within government, its agencies and powerful online corporate 'monopolies' such as Google and Facebook. However, there is a substantial and growing surveillance apparatus developing within health care and the social sphere, among others [1, 2].
The architecture of these developing e-surveillance systems is invariably central and is the principle reason why there is resistance to these activities in various parts of the general population and other institutions. However, non-central architectures are viable and may offer a safer approach to surveillance and the information society.

In this paper we describe a project that has used a distributed architecture to identify and survey a number of related populations shared between health care and social care within the Tayside region of Scotland, and involving three social work departments and one health care trust.

The objective of this project was to investigate early years of life, including pregnancy, from the perspective of both mother and child and the institutions they interact with. This required access to limited data from multiple sources within distinct jurisdictions and its successful integration and hosting at an independent third-party facility.

Although the scientific and administrative aspects of the project are interesting and important, they will not be discussed here, but will instead be the subject of a future paper. 


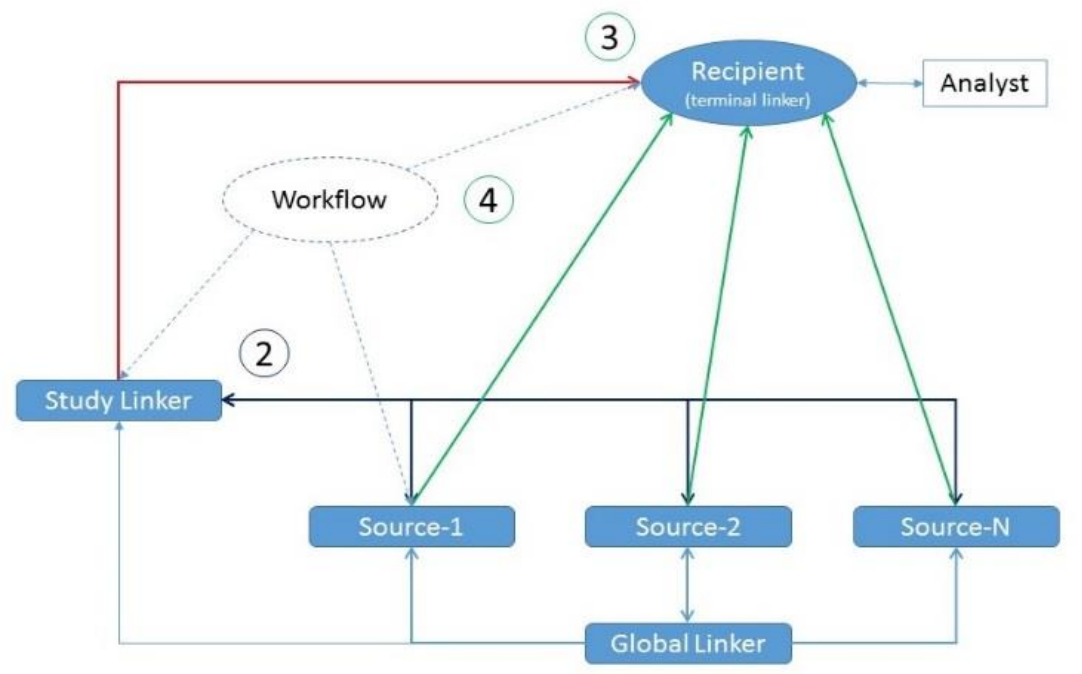

1

Figure 1: Proposed architecture for distributed data access and integration. 1) Global linkage, 2) Study linkage and preamble, 3) Terminal linkage at recipient, 4) Workflow

Here, we focus on some of the informational aspects of the project that require solutions, specifically: platform independent specifications for cohort definition, data selection and integration/harmonisation; assessment of data sources for content and quality, conceptual models employed, use of standards and technical readiness; methods of data access, selection, transformation and secure transport to a third-party; and cross-source data linkage and data joins using third parties.

In the present paper, some of these will be considered in a more democratic context and more open economic model.

\section{TECHNICAL APPROACH}

The approach we shall describe explicitly acknowledges the legally independent nature of most sources of data, as recognised in the concept of Data Control (DC) within the UK Data Protection Act 1998 [3]. In synthesising a new dataset for analysis within a distributed architecture, a number of information intermediaries are necessary to manage the selection, flow and combining of data into a semantically and structurally coherent whole for analysis.

In the present approach, these information intermediaries do not manipulate data, but do process information artefacts representing data subjects and the events associated with them for the explicit purposes of linkage and minimising the quantity of data released by sources. Data themselves, including derived data (from images for example), are transferred directly from data sources to third-party analysis facilities, where additional conditions of use and access to the integrated dataset are enforced.

\subsection{Data sources}

All professionally designed and deployed data sources will hold data in conformance with an information model and various standard or local coding systems. In the present approach, sources are assessed for their content and quality, and data are made selectable in discrete operations, which are formally described to the analyst community in terms of their content, coding and quality and are declared valid for recognised and formally specified purposes. The output of these operations can be raw data, derived data including simple aggregates, or information artefacts relating to subjects and events. This publication process and associated technical readiness involves effort on the part of source institutions and their personnel, where their rich knowledge of the data and context of collection is required for enabling data re-use.

For example, a source operation may provide a set of subject identifiers relating to a disease cohort constrained by age and type and provide guarantees about completeness, correctness and accuracy. Similarly, another source may provide operations relating to medication data for the set of subjects previously identified.

\subsection{Information intermediaries}

Most sources of data are not general purpose, but specialised to a lesser or greater degree. Therefore, most analyses will require data from more than one independent data source. Cross-source conditions for cohort and sub-cohort selection and temporal criteria may be specified, which necessitates the need for intermediaries to perform these kinds of operations if there is not to be wholesale outflow of data from sources. 
For example, hospital episode data in the UK holds information on discharge diagnoses and operations and procedures performed $[4,5]$, but nothing about laboratory test results or medication dispensed, which are held in other systems.

General sources of data, such as GP systems or clinical repositories, often hold copies of data from other systems in which the detailed context of the original data collection has not been preserved beyond where it came from. In addition, the quality of general sources is lower than that of specialised sources as the latter are likely to have better quality control, e.g. through adherence to guidelines.

As medicine and biology advance, new, specialised sources are constantly being generated and their use by generalists is likely to be a slow and flawed process. For example, genetic data in the UK, collected over a decade, is still only found within research systems and covers perhaps about $1 \%$ of the general population [6, 7]. Linkage of these genetic sources with healthcare sources is very important to the research community.

The present approach identifies and implements four information intermediaries: Global linker; intermediate linker or study linker; terminal linker or recipient; and workflow service. These are shown along with data sources in figure 1 .

\subsection{Workflow structure}

The creation of a dataset for analysis is a multi-step process. To achieve this, sources, study linkers and recipients publish operations that they can perform and these operations are parameterised and sequenced into a workflow. This workflow is constructed principally as a collaboration between analysts and data sources. This is true because the operations of global linker, study linker and recipient are well-defined and automatic and further consideration of their effect is generally not necessary.

However, sources and analysts may have a preference for which global linker, study linker and recipient they use on the basis of commercial or cohort sensitivity of the data being processed, as well as cost and speed of operations.

A workflow consists chiefly of two phases: a preamble phase and data export phase. The preamble phase is used to compute the minimum subsets of data to be exported by sources to the recipient, and also to coordinate pseudonymisation of subject identifiers by sources.

The export phase involves final selection of raw or derived data, pseudonymisation by sources, and secure transmission to the recipient.

\subsection{Linkage}

All data sources considered here hold data relating to living subjects and index that data with some kind of unique subject identifier (SID). Since most sources will use different SIDs, it is essential that another identifier is provided to sources that enables linkage. Linkage is defined (here) as the association of records in different sources to the same data subject, and subsequent allocation of the same subject identifier, or different identifiers with a known relationship.

At root, identification is a complex process involving many properties and attributes of a subject (such as name, DOB, gender and finger print). In the present approach, the global linker is provided for this purpose. If offers operations to both the sources and study linkers. For the former it will generate 'global' linkage identifier sets for incorporation into the source systems, each set being specific to one source and is re-usable. For the study linker it will make available the relationship between identifier sets when these are needed by a specific study linker during workflow.

When a source is globally linked, the global linker's matching engine will match the subjects' properties and attributes to its reference database. In the present project, a self-contained linkage engine (reference database and software) is placed within the jurisdiction of a source where it is physically secured by the source. The engine is electronically secured by the global linker. In a global linkage operation, which can be requested by a source at any time, staff from the global linker and source meet to enable access to the engine and performance of the matching operation.

Subject attributes and properties, such as those suggested above, are moved as a file from the source system to the engine using physical media. The file is in non-executable format such as ANSI text and the engine and source systems are configured to accept only this type of file on the media. These source-specific subject details are matched to the reference database and an identifier allocated and written back to the output file for incorporation into the source system. The global linker's engine becomes a permanent feature within a source's jurisdiction and updates to the reference database are also incorporated using physical media which are destroyed after use.

This process offers significant advantages to the source:

1) subject identification data never leaves the source jurisdiction, and

2) the source's network and systems are not exposed to the engine. 
The engine itself is protected through its electronic security and tight restrictions on the physical media.

In this way, the global linker can distribute a linkage reference to a group of sources (known as a linkage group).

Because each source gets its own reference identifier set, sources within a linkage group cannot link data for themselves (using the identifier sets), and the method provides a basis for strong governance of data sharing.

\subsection{Cross-platform data joins}

When a source identifies a cohort of interest under the direction of the workflow, the identifiers for that cohort are sent to the study linker. Since the study linker has the right to request the relationships between identifier sets from the global linker, the study linker is able to translate identifiers between sources and can perform logical operations on multiple cohorts from different sources or provide a cohort to any source as a filter for data exports and other logical operations applied at source. The information artefacts transferred from a source to a study linker can also include event timing as well as subject identifier to permit relative temporal constraints to be applied across sources.

In this way, one or more study linkers can assist in arranging for complex selections of data among independent sources and minimise the data that a source has to release to the recipient (figure 1).

\subsection{Study linkage}

Once the final cohorts have been generated from the pre-amble phase they can be passed to the relevant sources to provide a filter for data export to the recipient. In doing this the study linker generates random numbers to pair up with the reference identifiers for a source. When data is exported the data is filtered using the reference identifier, but exported to the recipient using the related random identifier. This change of linkage is known as the study linkage and ensures that the recipient cannot link data from different studies (at least in a trivial manner).

\subsection{Terminal linkage}

When the recipient receives data from multiple sources for the same study the data are identified by the random numbers, which are different for each source.

The relationships between the random numbers for each source is known to the study linker and this set of random identifier tuples is sent directly to the recipient from the study linker.
The recipient now has the means to link the data from the various sources into a final dataset with a new identifier allocated to each tuple (running from 1 to $n$ ). This change of linkage is known as the terminal linkage. Note that data received by the recipient can be inspected by analysts before the terminal linkage is authorised adding further degrees of safety.

These changes of linkage allow excellent governance if it is necessary to track analysed data back to the source and subject. To track back to a subject from the terminal linkage ID, the recipient, study linker and source must be involved. This is also the route for enquiries related to the provenance and quality of source data when the integrated dataset contains conflicts.

\subsection{Advantages of the mechanism}

The method involves significant advantages in terms of democratic control - all parties are independent. Data controllers remain in control - all other parties are processors, even the recipient. There are no preferential controllers arising from institutional importance or size. Data releases are minimised by use of the preamble reducing exposure risk for subject data. All source operations incorporate data quality guarantees and might be suitable for contractual obligations within an open information economy. Analysts, data sources, linkers, recipients and workflow services can freely associate on the basis of cost, quality, efficiency, effectiveness and trust. Because there are multiple, independent witnesses to all operations, external oversight can be very effective and could include oversight by data subjects.

Finally, no record of operational activities is maintained by the study linker; a distributed record of all activities sits with the sources and these can be integrated at any time for, for example, regulatory purposes. This is important as the study linker can, in theory, make inferences during its operations about subject data. No memory is retained of this. In theory, patients can also make (automated) requests to both sources and recipients to achieve a consistent view of the use of their data. Finally, the method is scalable and can be simultaneously implemented at local, national or international scales for the same source.

\section{EVALUATION}

The method was evaluated using a study investigating the relationships between children and mothers in their early years, including pregnancy, and the health care and social care institutions they interact with. 
Two systems and a clinical repository were identified in health care, and a single system was identified in each of three social work departments (two proprietary and one in-house). These sources were analysed and data operations identified that provide useful content and sufficient quality. Sources were then globally linked using a reference database derived from the NHSiS CHI for Tayside [8] as this covers over $96 \%$ of the population. Workflows were developed for a number of specific scenarios, which defined cohorts of interest. Workflows were then run and following the preamble the minimum required data transferred directly to the TASC Safe Haven as recipient [9].

The dataset was analysed for correctness of linkage. A full analysis will be reported in due course, where the readiness of sources to share data will be presented, and will include assessments of completeness, accuracy, correctness, data harmonisation, data availability, and resource use.

It is not possible to discuss these issues here owing to limited space. However, the method has specific recommendations for data harmonisation and quality.

\section{CONCLUSION}

The method successfully synthesised longitudinal datasets from the health and social care sectors in relation to cohorts of interest. It did so in a manner which offers significant safety improvements over centralised methods. The method should be considered in the context of broader objectives of a more democratic approach to controlling information flows and the need to build an inclusive information economy in which the data sources, analysts and subjects play a significant role. Data sources need to find the time and resources to fulfil this role.

\section{REFERENCES}

[1] Farr Institute E-Infrastructure. http://www.farrinstitute.org/5_EInfrastructure.htm I (24-12-2014)

[2] ISD Scotland. http://www.isdscotland.org/Products-andServices/ (24-12-2014)

[3] Data Protection Act 1998. http://www.legislation.gov.uk/ukpga/1998/29/cont ents (24-12-2014)

[4] Hospital Episode Statistics. http://www.hscic.gov.uk/hes (24-12-2014)

[5] Hospital Records Data Monitoring. http://www.isdscotland.org/Products-andServices/Hospital-Records-DataMonitoring/index.asp?Co=Y (24-12-2014)

[6] UK Biobank. http://www.ukbiobank.ac.uk/

[7] Wellcome Trust Case Control Consortium. http://www.wtccc.org.uk/ (24-12-2014)

[8] NHSiS CHI. http://www.scot-shiptoolkit.org.uk/roles-and-responsibilities/chiag (24-12-2014)

[9] Tayside Academic Health Sciences Collaboration. http://www.tahsc.org/ (24-122014) 